\title{
Parada cardíaca em crianças
}

\section{Cardiac arrest in children}

\author{
Alessandra Kimie Matsuno
}

\begin{abstract}
RESUMO
Este texto apresenta uma revisão da epidemiologia, das causas, do diagnóstico e do manejo da parada cardíaca em crianças. Ao contrário do adulto, a parada cardíaca súbita de origem cardíaca é um evento raro na criança. Geralmente, é causada por insuficiência respiratória e/ou choque. Portanto, o reconhecimento precoce e o tratamento imediato destas condições podem evitar a parada cardíaca e melhorar o desfecho desses pacientes.
\end{abstract}

Palavras-chave: Parada Cardíaca. Criança. Diagnóstico. Tratamento.

\section{Definiçåo}

Ritmo de colapso, parada cardiopulmonar ou simplesmente parada cardíaca são situações clínicas caracterizadas pela interrupção da circulação do sangue causada por ausência ou ineficácia da atividade mecânica cardíaca.

\section{Incidência}

A parada cardíaca súbita em crianças é pouco comum. O que ocorre nas crianças, geralmente, é a parada cardíaca decorrente da progressão da insuficiência respiratória e/ou do choque, associada à hipoxemia e acidose, sendo bem menor a incidência de parada cardíaca por arritmias cardíacas na faixa etária pediátrica do que no adulto. O colapso súbito devido à fibrilação ventricular (FV) ou taquicardia ventricular (TV) sem pulso ocorre como manifestação clínica inicial em aproximadamente $5-15 \%$ de todas as paradas cardíacas pediátricas pré-hospitalares; esta incidência deve ser um pouco mais alta em pacientes acima de 12 anos. Nos pacientes internados que sofreram parada cardíaca, haverá um ritmo chocável (TV/ FV) em algum momento durante a tentativa de ressuscitação em aproximadamente $27 \%$ das crianças, sendo que $10 \%$ apresentarão estes ritmos como manifestação inicial. ${ }^{1,2}$

\section{Sobrevivência à alta hospitalar}

As taxas de sobrevivência estão relacionadas à localização onde ocorreu a parada cardíaca e o tipo de ritmo de colapso presente. A sobrevivência à alta hospitalar na parada cardíaca dentro do hospital é maior quando comparada à parada cardíaca fora do hospital, assim como a sobrevivência sem sequelas neurológicas. Nos últimos 30 anos, a sobrevivência dentro do hospital aumentou de 9 para $27 \%$, enquanto que a sobrevida em pacientes tratados fora do hospital se
Professora Colaboradora do Departamento de Puericultura e Pediatria da Faculdade de Medicina de Ribeirão Preto da Universidade de São Paulo
Correspondência:

Avenida dos Bandeirantes 3900 14049-900 / Ribeirão Preto - SP. matsuno@fmrp.usp.br

Artigo recebido em 11/04/2012 Aprovado para publicação em 20/06/2012 
mantém a mesma, 6\% (3\% para lactentes e 9\% para crianças e adolescentes). A sobrevivência também é maior quando o ritmo inicial é chocável (25\%-33\%) comparado ao ritmo não-chocável à apresentação (7\%-11\%). Entretanto, quando um ritmo chocável se desenvolve durante a parada cardíaca em crianças hospitalizadas, o prognóstico é pior (sobrevida de $11 \%$ vs. $27 \%$ com ritmo não-chocável). De qualquer forma, a sobrevida é baixa e, portanto, enfoque maior deve ser dado à prevenção, pelo reconhecimento das situações de emergência que potencialmente podem levar à parada cardíaca. ${ }^{1,3,4,5}$

\section{Apresentação da parada cardíaca}

De acordo com a etiologia, a apresentação da parada cardíaca em crianças pode ser de dois tipos: parada cardíaca hipóxica ou por afixia e parada cardíaca súbita. A parada hipóxica é o mecanismo fisiopatológico mais comum de parada cardíaca na criança e ocorre como evento terminal de hipóxia tecidual progressiva e acidose, secundárias à insuficiência respiratória, choque ou insuficiência cardiopulmonar. A parada cardíaca súbita em crianças é geralmente associada a arritmias cardíacas, especificamente a FV e a TV sem pulso. As causas de parada cardíaca estão relacionadas na Figura 1.

Qualquer que seja o evento inicial ou o processo patológico envolvido no tipo de parada, os pacientes desenvolvem falência cardiopulmonar imediatamente antes da parada cardíaca. A falência cardiopulmonar se caracteriza por oxigenação, ventilação e perfusão tecidual inadequadas, e clinicamente o paciente se apresenta cianótico, com respiração agônica ou irregular e bradicardia. O modelo de avaliação pediátrica permite que se reconheça e trate a falência cardiopulmonar imediatamente. A parada cardíaca é reconhecida por ausência de sinais da função cardiorrespiratória (sem movimentação, sem ventilação e sem pulso). Clinicamente, o paciente em parada cardíaca se apresenta com apneia ou respiração agônica, sem pulsos detectáveis e não responsivo (inconsciente). A monitorização do ritmo cardíaco não é mandatória para o reconhecimento clínico da parada cardíaca, mas serve para direcionar a terapêutica. ${ }^{6}$

Os ritmos de colapso ou de parada cardíaca são: assistolia, atividade elétrica sem pulso (AESP), FV e TV sem pulso. Enquanto que a assistolia e a AESP são os ritmos iniciais mais comumente observados na parada cardíaca em crianças abaixo de 12 anos, a FV e a TV são os ritmos iniciais observados em crianças que apresentam colapso súbito. ${ }^{7}$

\section{Identiflcação dos ritmos de colap- so no eletrocardlograma (ECG)}

- Assistolia: Ritmo de parada cardíaca associado à ausência de atividade elétrica detectável pelo ECG e representado por uma linha reta (Figura 2). Devese confirmar clinicamente este diagnóstico para descartar artefato ou derivações mal-fixadas do ECG. $^{8}$

- Atividade Elétrica sem Pulso (AESP): Qualquer atividade organizada observada no monitor cardíaco ou no ECG em paciente sem pulso central palpável; esta definição exclui FV, TV e assistolia. O ECG pode mostrar a presença de complexo QRS normal ou alargado, ou outras anormalidades como

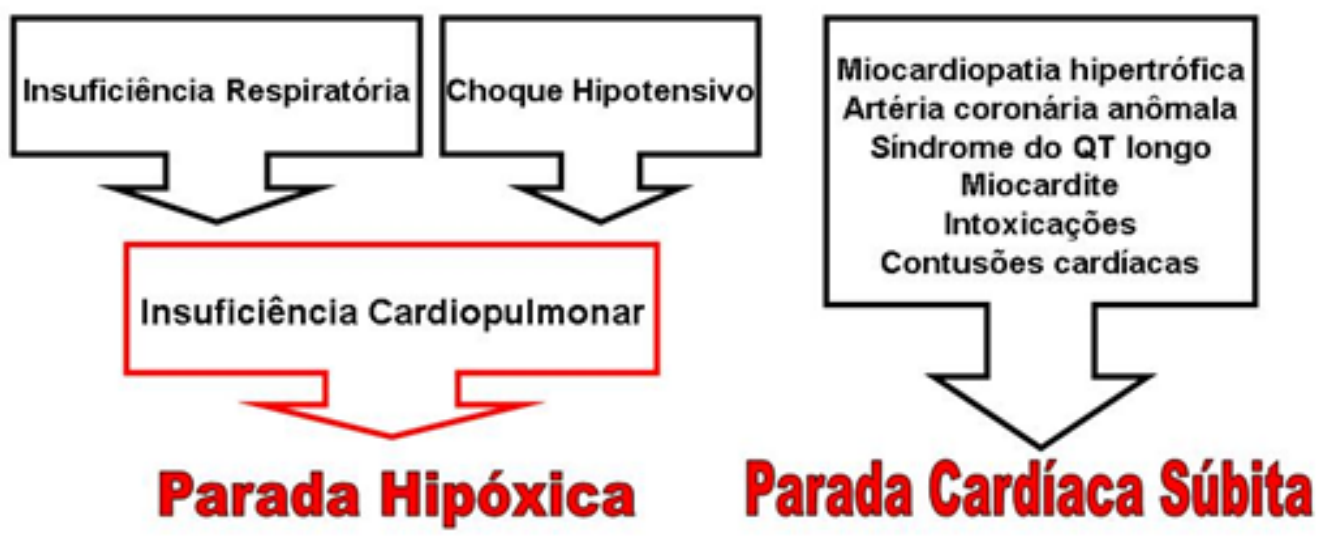

Figura 1: Tipos de apresentação e causas de parada cardíaca em crianças. 


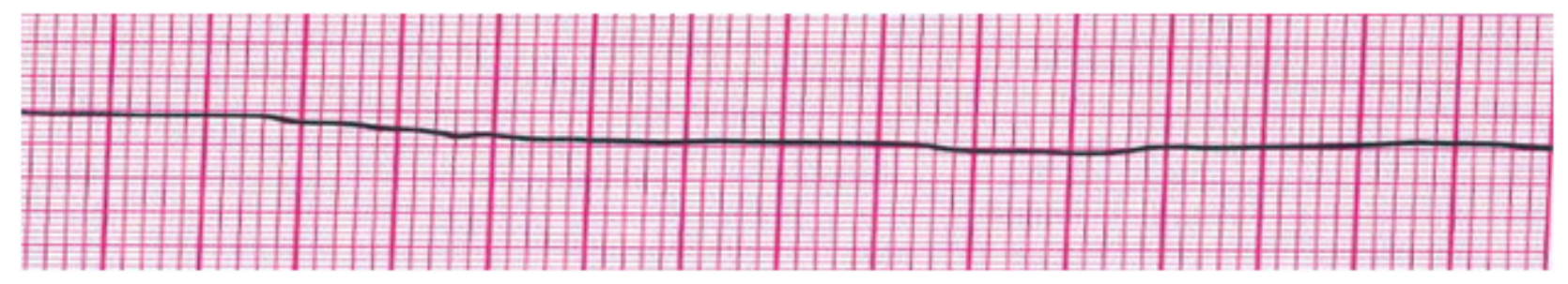

Figura 2: Assistolia

onda T com baixa ou alta amplitude, intervalo PR e QT prolongados e dissociação atrioventricular ou bloqueio cardíaco total. O ECG pode fornecer dados importantes para determinar a etiologia da parada cardíaca. Geralmente, quando o QRS é inicialmente normal, a AESP pode ter sido causada por problemas de curta duração, como hipovolemia ou hemorragia, embolia pulmonar maciça, pneumotórax hipertensivo ou tamponamento cardíaco. Por outro lado, a AESP com ritmo lento e com complexos QRS largos é observada em processos de maior duração, como hipóxia tecidual e acidose. AAESP pode ser causada por condições reversíveis e, portanto, durante a reanimação cardíaca deve-se sempre procurar e tratar suas causas. ${ }^{8}$

- Fibrilação ventricular: O coração não apresenta ritmo organizado, não tem contrações coordenadas e a atividade elétrica é caótica; portanto, o coração tremula e não bombeia sangue (Figura 3). A FV pode ser causada por doenças cardíacas, intoxicações, choques elétricos, acidentes por submersão e traumas, e geralmente se associa com colapso súbito. Os pacientes que apresentam FV ou TV sem pulso como ritmo de colapso inicial têm taxa de sobrevivência maior do que aqueles com assistolia e AESP, principalmente se o diagnóstico for precoce e o tratamento iniciado o mais rápido possível. Se houver demora no reconhecimento da FV ela pode evoluir para fibrilação de baixa voltagem, que apresenta um pior prognóstico com menor resposta ao tratamento (Figura 4). ${ }^{8}$

- Taquicardia ventricular (TV) sem pulso: Caracteriza-se por complexos QRS organizados e largos. Ao ECG, apresenta-se com frequência ventricular

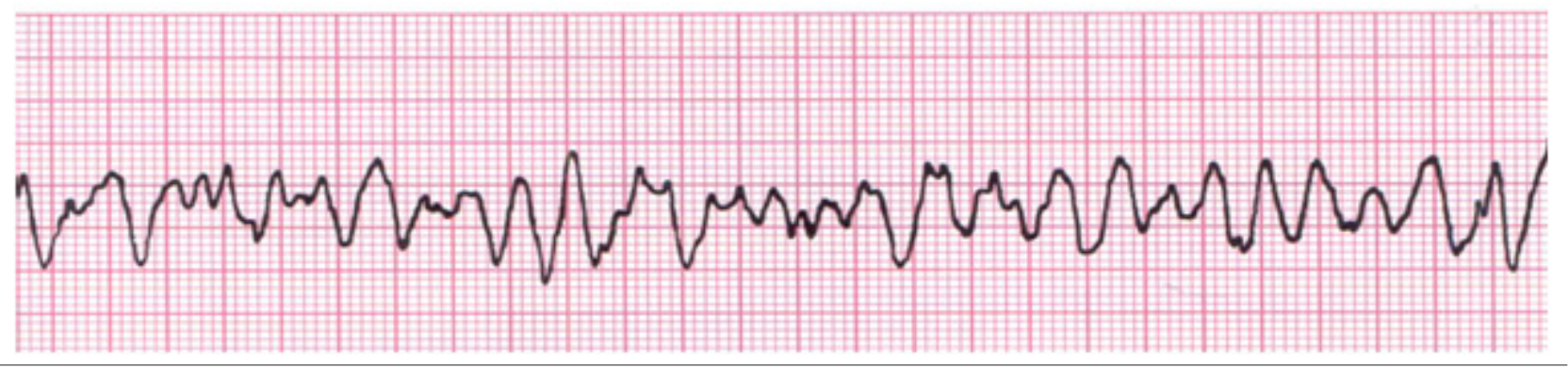

Figura 3: Fibrilação ventricular de alta voltagem.

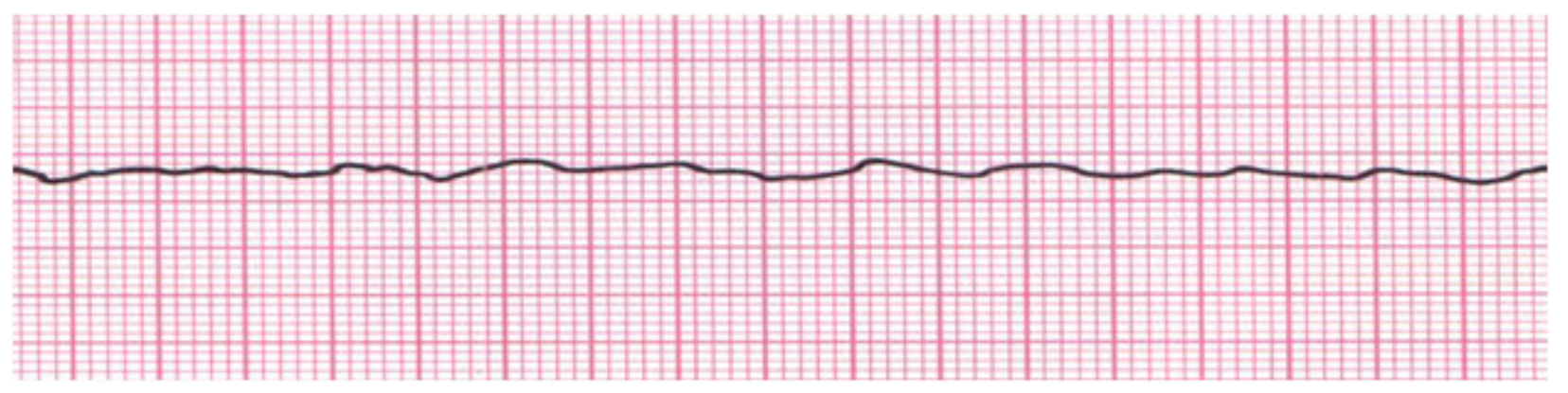

Figura 4: Fibrilação ventricular com atividade elétrica reduzida ou de baixa voltagem. 
de pelo menos 120/min, QRS > 0,08 segundos de duração, ondas $\mathrm{P}$ geralmente não identificadas, ou quando presentes, podem não estar correlacionadas ao QRS, e ondas T geralmente de polaridade oposta ao QRS. As causas podem estar associadas à doença cardíaca de base (ou pós-operatório de cardiopatia), síndrome do QT longo, miocardite, miocardiopatias, distúrbios hidroeletrolíticos (hiperpotassemia, hipocalcemia, hipomagnesemia) e intoxicações (antidepressivos tricíclicos e cocaína). A TV sem pulso pode ser monomórfica, quando os complexos QRS têm aparência uniforme (Figura 5) ou polimórfica, quando os complexos QRS não são uniformes (Figura 6). Um exemplo de TV polimórfica é a chamada torsades de pointes ("torção dos pontos"), caracterizada por uma alteração de polaridade e de amplitude dos complexos QRS, que parecem rodar ao redor de uma linha isoelétrica do ECG. As condições que predispõem à torsades de pointes incluem a síndrome do QT longo, hipomagnesemia, intoxicação por antiarrítmicos (Classe IA: quinidina, procainamida e disopiramida; Classe III: sotalol, amiodarona), intoxicação por antidepressivos tricíclicos, bloqueadores de canais de cálcio e fenotiazina. ${ }^{8}$

Em todos os ritmos de colapso é necessário, além do tratamento do ritmo, procurar as causas pro- váveis e tratá-las. Há um método mnemônico que ajuda a lembrar das causas mais comuns dos ritmos cardíacos de colapso. São os 6 Hs e os 5 Ts ${ }^{7}$ :

- Hipovolemia

- Hipóxia

- Hidrogênio (distúrbios do $\mathrm{H}^{+}$- o mais comum é a acidose)

- Hipo/hiperpotassemia

- Hipoglicemia

- Hipotermia

- Tóxicos (intoxicações em geral)

- Tamponamento cardíaco

- Tensão no tórax (Pneumotórax)

- Trombose coronariana

- Trombose pulmonar

\section{Tratamento dos ritmos de colapso}

A alta qualidade da ressuscitação cardiopulmonar (RCP) e a sistematização do atendimento são bases fundamentais para o sucesso do tratamento da parada cardíaca, tanto no suporte básico como no suporte avançado de vida. ${ }^{7}$

\section{Suporte básico de vida}

O suporte básico de vida (suporte dado fora do hospital) inclui o reconhecimento dos sinais de parada

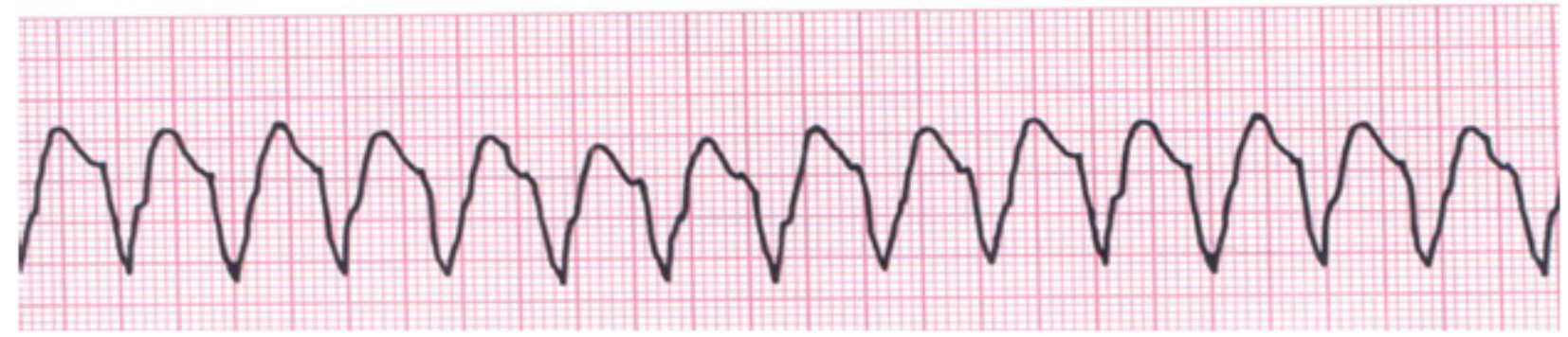

Figura 5: Taquicardia ventricular monomórfica.

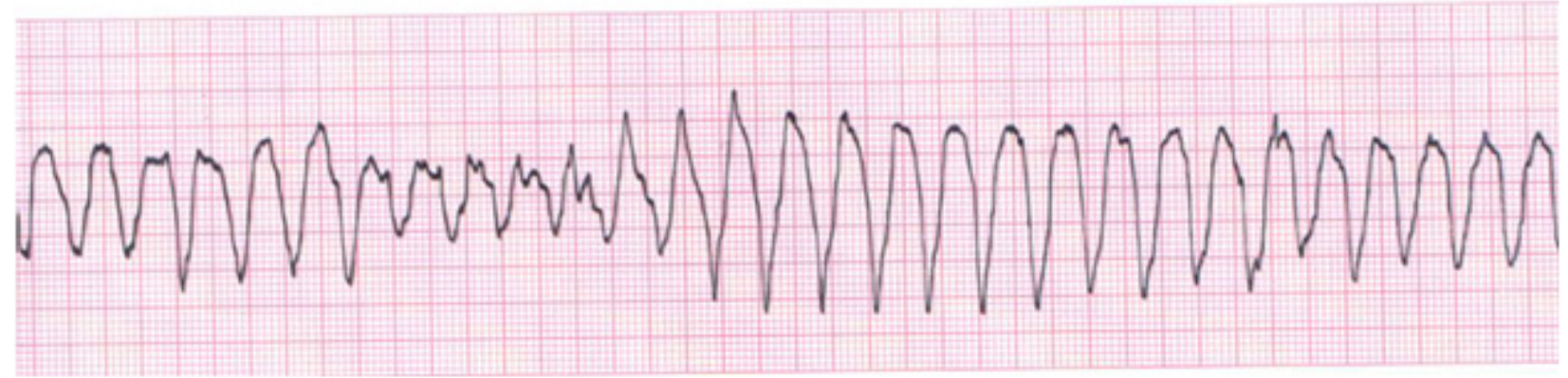

Figura 6: Taquicardia ventricular polimórfica. 
cardíaca, a realização da RCP de qualidade e o uso da desfibrilação com desfibrilador externo automático (DEA). Na recomendação de 2010, os profissionais de saúde e leigos devem considerar no atendimento das crianças as seguintes faixas etárias: lactentes como aqueles menores que 1 ano e crianças como aquelas de 1 ano até a idade do início da puberdade (adolescência) definida como desenvolvimento de mamas nas meninas e pelos axilares nos meninos; a partir daí, o tratamento é igual ao ofertado aos adultos. ${ }^{3}$

\section{Considerando o cenário fora do hospital}

Cenário 1. Se um paciente pediátrico estiver com sinais de parada cardíaca (inicialmente inconsciente, em apneia ou com respirações agônicas) e a parada não foi testemunhada, deve-se realizar a RCP, da seguinte maneira: "CAB", ou seja: C - checar o pulso, se ele não for palpável ou for difícil a sua palpação (não demorar mais do que 10 segundos com as tentativas de sentir o pulso) iniciar imediatamente a massagem cardíaca e, subsequentemente, faz-se o A e o B.

A- Posicionar e garantir a abertura das vias aéreas, usando manobra de extensão do pescoço e elevação da mandíbula.

B- Fornecer duas ventilações e continuar a massagem cardíaca, conforme relação descrita a seguir.

A relação deve ser de 30 compressões: 2 ventilações, se houver um socorrista; caso mais um socorrista esteja ajudando, esta relação é de 15:2 em crianças. Estudos demonstraram que a qualidade da massagem cardíaca é imprescindível para o retorno da circulação; portanto, a partir das recomendações das diretrizes de 2005 e 2010 tem-se dado ênfase à qualidade da técnica da massagem, que deve ser feita da seguinte forma: ${ }^{9}$

- Local: Terço inferior do esterno, evitando o apêndice xifoide ou as costelas. Nas crianças abaixo de 1 ano, o local recomendado é $1 \mathrm{~cm}$ abaixo da linha inter-mamilar (Figura 7) e nas crianças acima de 1 ano, o local recomendado é na linha inter-mamilar (Figura 8$).^{3}$

- Profundidade: No mínimo, 1/3 do diâmetro anteroposterior ou $4 \mathrm{~cm}$ em lactentes e $5 \mathrm{~cm}$ em crianças. ${ }^{3}$
- Técnica: Em crianças abaixo de 1 ano, a melhor técnica é a aquela que usa os dois polegares com as mãos circundando o corpo; se houver apenas um profissional, pode-se tentar a técnica dos dois dedos (segundo e terceiro dedos da mão). Em crianças maiores, deve-se realizar a massagem com a palma de uma mão (região tênar e hipotênar) na região intermamilar ou com a segunda mão por cima.

- Frequência da compressão: 100/min.

- Relação compressão: ventilação: Como dito anteriormente, deve ser de acordo com o número de socorristas, o conhecimento do socorrista e a faixa etária do paciente. Se os socorristas forem leigos (1 ou 2) ou se for um profissional de saúde sozinho, esta relação será sempre 30:2 para todas as faixas etárias. Se forem 2 socorristas e um deles for profissional de saúde, esta relação

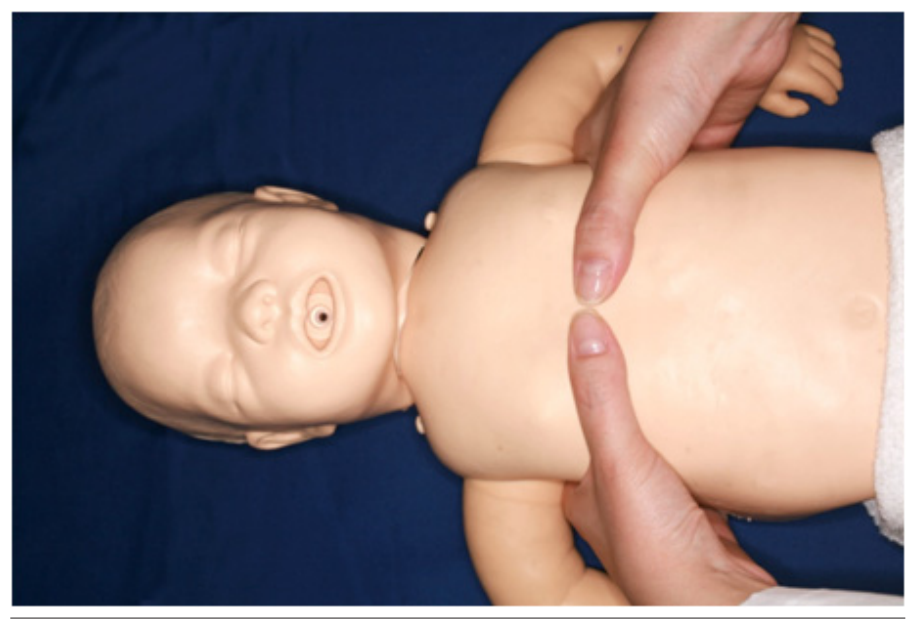

Figura 7: Técnica de massagem cardíaca em lactentes.

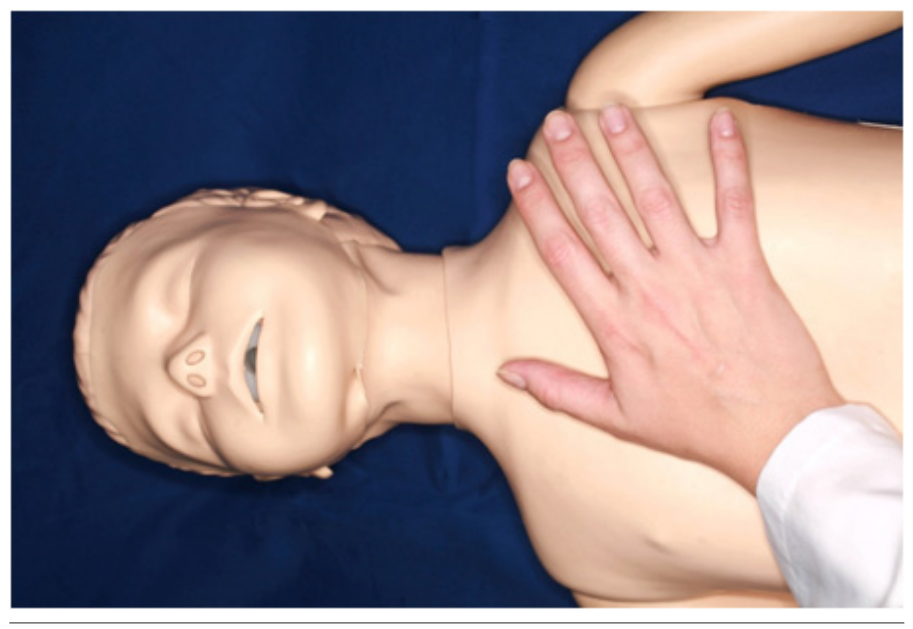

Figura 8: Técnica de massagem cardíaca em crianças maiores de 1 ano. 
será 15:2 até adolescentes, e 30:2 acima desta faixa etária. A quantidade maior de ventilações na faixa etária pediátrica decorre do fato de que a hipóxia é causa comum de parada cardíaca nestes pacientes.

- Interromper a massagem cardíaca, o mínimo possível, apenas durante a ventilação, pois estudos demonstram que toda a vez que se para a massagem cardíaca, ao reiniciá-la, demora-se um tempo para chegar novamente em uma pressão ótima para a perfusão coronariana (necessária para o retorno da circulação). ${ }^{3}$

- Trocar as funções de compressor e fornecedor da ventilação a cada 2 minutos, pois haverá um cansaço natural do compressor, prejudicando a qualidade da massagem. Caso haja apenas um socorrista neste cenário (colapso súbito não testemunhado), deve-se fazer um ciclo de RCP (massagens cardíacas e ventilações) por 2 minutos antes de chamar o serviço de emergência ou de conseguir um DEA; se houver mais de uma pessoa, deve-se pedir a ela que chame o serviço de resgate ou que busque o DEA imediatamente, após verificar que o paciente está inconsciente. ${ }^{3}$

Cenário 2. Se o paciente tiver um colapso súbito testemunhado e se encontrar com sinais de parada cardíaca, antes de iniciar a RCP na sequência citada anteriormente, deve-se acionar o serviço de emergência ou conseguir um DEA, se você estiver sozinho. O DEA é programado para avaliar o ECG da vítima e verificar se o ritmo é chocável ou não; se for um ritmo chocável, ele orienta o socorrista em relação ao momento ideal para dar o choque por meio de mensagens sonoras e visuais. Nas novas diretrizes de 2010 sobre o DEA, recomenda-se utilizar um atenuador de carga elétrica pediátrico para crianças de 1 a 8 anos, mas na ausência deste, pode ser utilizado o DEA com carga padrão. Em crianças menores de 1 ano, recomenda-se em primeiro lugar a utilização do desfibrilador manual, mas na ausência deste, pode ser utilizado o DEA com atenuador de carga elétrica pediátrico e se caso este também faltar, pode-se usar o DEA com carga padrão. Esta mudança ocorreu, pois não se sabe a carga mínima eficaz nem a carga máxima prejudicial na desfibrilação das crianças, mas sabese, por estudos de modelos animais pediátricos, que cargas acima de $4 \mathrm{~J} / \mathrm{kg}$ (até $9 \mathrm{~J} / \mathrm{kg}$ ) não têm efeitos adversos significativos. ${ }^{3}$

Observação: Nos casos de parada cardíaca fora do hospital, se o socorrista for leigo, assim que detectar inconsciência, ausência de respiração ou respiração agônica da vítima, ele deverá iniciar a massagem cardíaca sem verificação do pulso.

\section{Suporte avançado de vida}

No cenário intra-hospitalar, provavelmente o atendimento não será feito por um único profissional. Portanto, assim que se detectar que o paciente apresenta parada cardíaca, deve-se pedir para que se traga o carrinho de emergência com o desfibrilador e a monitorização e, enquanto isso, inicia-se a RCP. A meta das intervenções terapêuticas é o retorno da circulação espontânea, definido como a restauração de ritmo cardíaco espontâneo (ao monitor é o aparecimento de atividade elétrica organizada) com perfusão (pulsos centrais palpáveis e pressão arterial mensurável). O tratamento dos ritmos de colapso inicia-se sempre com o "CAB":

C- Se houver ausência de pulso central, inicia-se a massagem cardíaca de alta qualidade seguindo as mesmas recomendações anteriormente expostas e depois, faz-se o A e o B. ${ }^{7}$

A- Abertura de vias aéreas pelo posicionamento correto da cabeça e aspiração.

B- Oferta de oxigênio, que neste caso será através da bolsa-valva e máscara, pois o paciente em parada cardíaca não apresenta respiração espontânea ou quando apresenta, ela é totalmente ineficaz (respiração agônica). A intubação orotraqueal deverá ser feita assim que possível, quando diminuir a efetividade da ventilação com bolsa-valva e máscara. $\mathrm{O}$ momento adequado para a intubação dependerá da experiência do socorrista, pois ela deve ser feita rapidamente e durante a sua realização, é necessário parar as manobras de RCP.

Se dois socorristas estiverem atendendo crianças até a adolescência, a relação compressão: ventilação deve ser 15:2 e em adolescentes e adultos, 30:2. Após a intubação, a maneira como se ventila e massageia muda, deixando de ser sincronizada e passa a ser 8-10 ventilações por minuto (6 segundos entre uma ventilação e outra) e a massagem se torna contínua sem interrupções. Esta mudança se deve a estudos que mostram que durante a parada cardíaca, o fluxo sanguíneo para os pulmões é de somente $25 \%$ a $33 \%$ do normal; isso significa que durante a ressuscitação é necessário menos ventilação para satisfazer a perfusão. 
As manobras de abertura de vias aéreas e ventilação, principalmente na parada cardíaca em crianças, cuja principal causa é a hipóxia, são extremamente importantes e não podem ser retardadas, pois um estudo recente com pacientes pediátricos mostrou que há melhor desfecho, nestes casos, quando se combina a massagem cardíaca com a ventilação do que apenas com a massagem cardíaca. Teoricamente, o início da ventilação não poderá demorar mais que 18 segundos após o início da massagem com um socorrista e se forem dois socorristas, este tempo deverá ser ainda menor (18 segundos é o tempo que o socorrista leva para aplicar 30 compressões, a 100/min).

$O$ acesso venoso deve ser estabelecido assim que se detectar uma situação de emergência. A prioridade para o estabelecimento do acesso vascular deve ser na seguinte sequência: intravenoso, intraósseo e endotraqueal. ${ }^{8}$

- Veia periférica: Este acesso é preferível como primeira tentativa, pois além de ser mais fácil de ser obtido que o acesso central (que precisa de habilidades específicas), durante a sua aquisição não é necessário interromper as manobras de RCP. Caso o paciente já esteja com um acesso central antes do início das manobras de RCP, esta via deve ser utilizada para medicações, porque possibilita início de ação mais rápido e concentrações mais elevadas do que a veia periférica.

- Via intraóssea: Caso não se consiga o acesso periférico, deve-se dar preferência à via intraóssea (IO), que pode ser estabelecida em qualquer faixa etária, inclusive nos pacientes adultos. Constitui acesso fácil, frequentemente obtido em 30 a 90 segundos, onde podem ser feitos quaisquer tipos de medicação ou de fluido administrados normalmente por via endovenosa, pois a canulação IO fornece um acesso não colapsável para o plexo venoso da medula óssea.

- Via endotraqueal: Deve ser utilizada apenas na impossibilidade de obtenção da via intravenosa ou intraóssea, pois ela possui algumas restrições: 1) Só podem ser administradas medicações lipossolúveis como atropina, naloxona, epinefrina e lidocaína ("ANEL"); 2) a dose ideal da maioria das medicações é desconhecida; 3) a absorção das medicações pela árvore traqueobrônquica é imprevisível e, portanto, seus níveis e efeitos também serão imprevisíveis; 4) a administração endotraqueal das medicações resulta em níveis sanguíneos inferiores quando se compara com as mesmas doses administradas por via intravenosa ou intraóssea; 5) a dose recomendada de epinefrina por via endotraqueal é dez vezes maior que aquela administrada por via intravenosa e para as outras medicações, é duas a três vezes maior. A técnica de administração consiste em instilar as drogas no tubo traqueal diluídas em $5 \mathrm{ml}$ de solução fisiológica, aplicando-se 5 ventilações com pressão positiva após a instilação.

Após a identificação do ritmo pelo ECG, devese iniciar o tratamento apropriado de acordo com o tipo de ritmo (chocável ou não-chocável).

\section{Tratamento dos ritmos não-chocáveis}

Constituem ritmos não-chocáveis a assistolia e a AESP. Assim que se detectar a ausência de pulso central, deve-se iniciar a massagem cardíaca de alta qualidade e administrar epinefrina durante as manobras de RCP, ou seja, enquanto se faz a massagem cardíaca. A cada 5 ciclos ou 2 minutos de RCP, verifica-se o ritmo. A dose de epinefrina por via intravenosa ou intraóssea é de $0,01 \mathrm{mg} / \mathrm{kg}$ ou $0,1 \mathrm{~mL} / \mathrm{kg}$ da solução 1:10.000 (1 ampola diluída para $10 \mathrm{~mL}$ ), podendo ser repetida em intervalos de 3 a 5 minutos. A dose pela via endotraqueal deve ser 10 vezes maior, ou seja, $0,1 \mathrm{mg} / \mathrm{kg}(0,1 \mathrm{~mL} / \mathrm{kg}$ da solução $1: 1.000)$. Não existem benefícios quanto à sobrevivência que justifiquem o uso de altas doses de epinefrina $(0,1 \mathrm{a} 0,2 \mathrm{mg} / \mathrm{kg})$; isto pode ser até mesmo prejudicial, principalmente em casos de parada hipóxica. ${ }^{10}$ No entanto, o uso de altas doses pode ser considerado em algumas situações especiais, como na intoxicação por $\beta$-bloqueadores. Ressalta-se que a epinefrina é a única droga indicada na RCP dos ritmos não-chocáveis em crianças. Após iniciar a massagem cardíaca, devem-se assegurar as vias aéreas e fornecer oxigenação rapidamente. É necessário também tratar as condições reversíveis associadas a estes ritmos (pensar nos $6 \mathrm{Hs}$ e 5Ts). Os ritmos assistolia e AESP nunca devem ser tratados com choque elétrico. ${ }^{3}$

\section{Tratamento dos ritmos chocáveis}

Constituem ritmos chocáveis a FV e a TV sem pulso. Inicialmente, faz-se a sequência de massagem cardíaca, abertura das vias aéreas e oxigenação apropriada. ${ }^{7}$ Após 5 ciclos ou 2 minutos de RCP, verificase o ritmo.

Ao chegar o desfibrilador, que no cenário intrahospitalar provavelmente será um desfibrilador ma- 
nual, faz-se a preparação e a aplicação do choque da seguinte forma: ${ }^{7,8,11}$

- Escolha das pás adequadas: Para crianças < 10 $\mathrm{kg}$ ou menores de 1 ano recomendam-se pás pequenas ou pediátricas, enquanto que para crianças $>10 \mathrm{~kg}$ ou maiores de 1 ano, recomenda-se o uso de pás grandes ou de adultos. Os locais apropriados para a colocação das pás são o lado superior direito do tórax, abaixo das clavículas e à esquerda do mamilo esquerdo, na linha axilar anterior (no ápice do coração); as pás devem estar distantes pelo menos $3 \mathrm{~cm}$ e nunca devem se tocar. Caso só estejam disponíveis pás de tamanho grande e seja necessário desfibrilar uma criança menor de $10 \mathrm{~kg}$, deve-se utilizar uma posição alternativa, que consiste em colocar uma pá na região anterior do tórax (à esquerda do esterno) e a outra posterior, nas costas.

- Podem-se utilizar pás autoadesivas ou convencionais. Caso sejam utilizadas pás convencionais, deve-se colocar gel, pasta ou creme condutor entre as pás e a pele do paciente para reduzir a impedância transtorácica. As pás NÃO DEVEM SER embebidas em solução salina ou gel para ultrassom, muito menos em compostos à base de álcool.

- A desfibrilação não deve ser sincronizada.

- Escolher a carga: Inicialmente deve ser de $2 \mathrm{~J} / \mathrm{kg}$.

- Colocar as pás na posição adequada.

- Carregar o aparelho.

- Para garantir a segurança da desfibrilação, devese realizar uma verificação visual do paciente e da equipe de ressuscitação, avisando com voz firme e forte antes de cada choque: "Vou aplicar o choque no três: um, estou fora (afastado do contato físico com o paciente, com a maca e com outro equipamento; dois, vocês estão fora; e três, oxigênio fora (que deve estar fora da área ao redor do tórax durante a desfibrilação)." Há outras situações especiais, além do uso de oxigênio, que exigem cuidados durante a desfibrilação: se o paciente estiver molhado, ele deve ser secado; na presença de marca-passo, deve-se colocar as pás a $2,5 \mathrm{~cm}$ de distância dos dispositivos; e na presença de medicamentos em adesivos transdérmicos, as pás não devem ser colocadas diretamente sobre estes adesivos (se necessário, devem ser removidos).

- O tempo desde o preparo do desfibrilador até a administração do choque não deve ultrapassar 10 segundos; enquanto se prepara a desfibrilação, devese continuar a RCP. Após aplicação do choque, reiniciam-se imediatamente as manobras de ressuscitação, começando pela massagem cardíaca, que deve ser feita por dois minutos. Subsequentemente, verifica-se o pulso central e o ritmo, e se este ainda for chocável, prepara-se para um novo choque com $4 \mathrm{~J} / \mathrm{kg}$. A partir do terceiro choque, esta carga será de $4 \mathrm{~J} / \mathrm{kg}$, ou poderá ser aumentada até $9 \mathrm{~J} / \mathrm{kg}$, devendo-se considerar a administração de epinefrina.

- Administração de medicamentos: Assim que se detecta que o ritmo chocável não reverteu após o primeiro choque e dois minutos de RCP, deve-se administrar epinefrina. Embora não tenha sido determinado um momento exato para a administração de epinefrina, recomenda-se que ela deva ser feita durante as compressões torácicas ou imediatamente antes do início das compressões, pois a droga deve "circular" para agir. As doses da epinefrina são as mesmas utilizadas no tratamento dos ritmos não-chocáveis.

- Outras medicações: Logo após o segundo choque, inicia-se a massagem cardíaca e faz-se a RCP por dois minutos, verificando-se novamente o pulso e o ritmo. Se a TV sem pulso ou a FV ainda persistirem, prepara-se um novo choque com $4 \mathrm{~J} / \mathrm{kg}$, podendo-se usar novamente a epinefrina ou algum antiarrítmico como amiodarona, lidocaína ou sulfato de magnésio (para torsades de pointes), cujas doses recomendadas são: Amiodarona $5 \mathrm{mg} / \mathrm{kg}$ IV ou IO (dose máxima única $300 \mathrm{mg}$ ); lidocaína 1mg/ $\mathrm{kg}$ IV ou IO, sendo que por via endotraqueal deve ser feita 2-3 vezes esta dose; sulfato de magnésio 25 a $50 \mathrm{mg} / \mathrm{kg}$ IV ou IO, dose única máxima de $2 \mathrm{~g}$. $\mathrm{O}$ algoritmo da parada sem pulso encontra-se na Figura 9.

\section{Motivos da mudança da abordagem "ABC" para "CAB"}

Durante a parada cardíaca, a RCP de alta qualidade e, particularmente as compressões torácicas são essenciais para enviar fluxo sanguíneo para os órgãos vitais e, desta forma, fazer com que retorne a circulação espontânea. Os argumentos a favor de começar pela massagem cardíaca são os seguintes: 1) A maioria das paradas cardíacas ocorre em adultos e o ritmo mais frequente é a fibrilação ventricular, em que a compressão torácica é mais importante que a ventilação; estes casos têm um melhor desfecho quando a massagem cardíaca se inicia o mais precocemente possível. 2) Para todos os socorristas é mais fácil e rápido iniciar com a massagem cardíaca, do que ini- 


\section{Algoritmo de Parada sem Pulso do PALS}

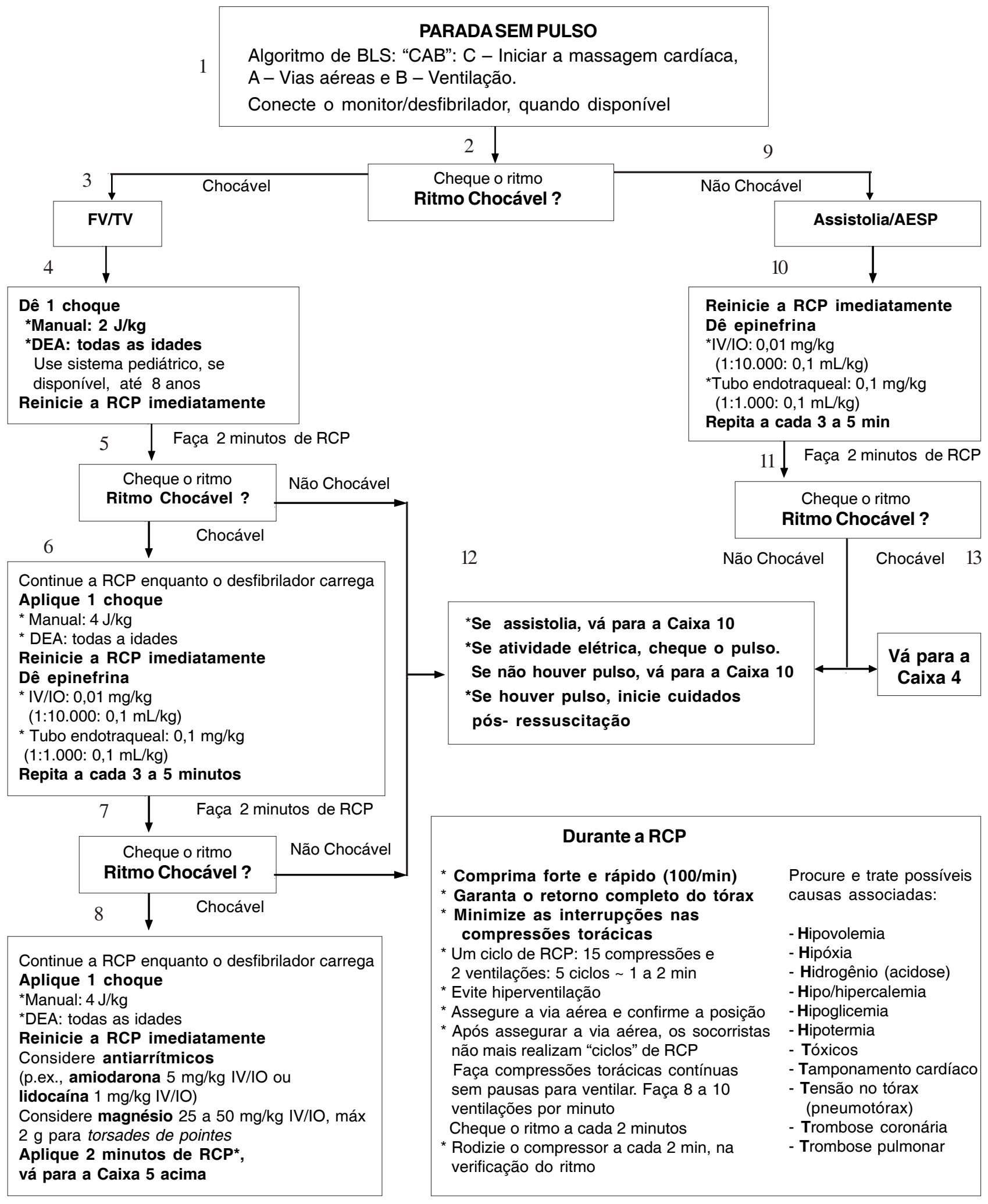

Figura 9: Algoritmo de Parada sem Pulso do PALS 
ciar com as manobras de abertura de vias aéreas e técnicas de ventilação; em muitos casos, houve retardo do início da massagem em decorrência de dificuldade técnica nos primeiros passos. 3) Ainda não se sabe se para as crianças, esta sequencia " $\mathrm{CAB}$ " tem diferenças em relação à abordagem antiga "ABC", mas ela está sendo recomendada para simplificar o treinamento do socorro às vítimas de parada cardíaca e amplificar a RCP adequada nas vítimas de colapso súbito, melhorando, desta forma, o seu prognóstico. . $7,12,13^{2}$

\section{Quando parar a RCP?}

Ainda não há consenso sobre o momento em que se devem interromper os esforços de ressuscitação, tanto nos ritmos chocáveis como nos não-chocáveis. A decisão de parar a RCP é influenciada pela causa provável da parada, pelos recursos disponíveis e pela probabilidade da presença de quaisquer condições reversíveis. Normalmente, o tempo de RCP varia de 20 a 30 minutos. Esforços prolongados devem ser feitos em lactentes e em crianças com FV ou TV recorrente ou refratária, toxicidade por drogas (até que se possa fazer o tratamento toxicológico adequado) e hipotermia (até que as medidas de reaquecimento adequado tenham sido realizadas). A evolução da parada cardíaca em pacientes pediátricos é geralmente ruim. Assim, o pronto reconhecimento dos ritmos de colapso e a RCP imediata e de alta qualidade, além da rápida correção dos fatores contribuintes oferecem melhores chances de sobrevivências para estes pacientes. ${ }^{14}$

\section{Casos clínicos}

\section{Caso 1}

Criança de 7 meses de vida, previamente saudável, é encontrada pela mãe no berço inconsciente e cianótica, após a mamada da madrugada. A criança chega à sala de emergência inconsciente, cianótica, em apneia e com pulsos centrais ausentes.

\section{Pergunta-se: Quais são o diagnóstico e a con-} duta?

Resposta: O diagnóstico é de parada cardiorrespiratória. A conduta é inicialmente pedir para trazer a monitorização / desfibrilador e iniciar o "CAB":

C. Quando os pulsos centrais estão ausentes, deve-se iniciar massagem cardíaca de alta qualidade, comprimindo forte e rápido (100 movimentos por min), garantindo o tempo de recolhimento e minimizando as interrupções.

A. Abrir as vias aéreas, posicionando o paciente com coxim sob os ombros, entre as escápulas e aspirar as vias aéreas (houve saída de grande quantidade de leite).

B. Fornecer oxigenação, que neste caso, deve ser sob a forma de ventilação com pressão positiva com bolsa-valva e máscara. A relação da compressão: ventilação, com dois socorristas, deve ser de 15:2. Após garantir as vias aéreas com a intubação traqueal, a ventilação não deve ser sincronizada, que deve ser 8 -10/min e a massagem cardíaca deve ser contínua. Assim que chegar o monitor, deve-se analisar o ritmo, que neste caso se encontra da seguinte forma:

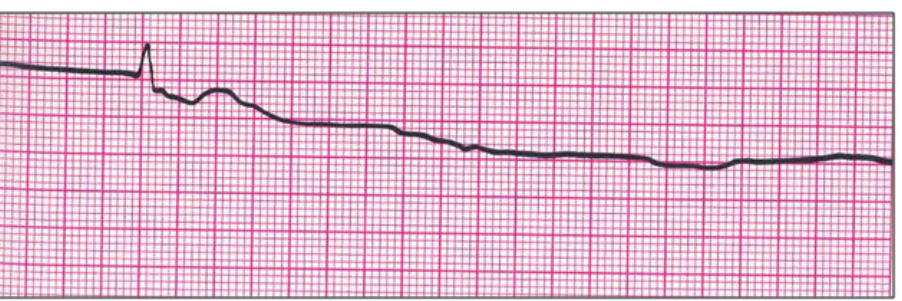

Pergunta-se: Quais são o diagnóstico e a conduta?

Resposta: O diagnóstico é assistolia e a conduta é manter RCP com massagem cardíaca e ventilação. Após o estabelecimento de acesso venoso ou intraósseo, deve-se administrar epinefrina $0,01 \mathrm{mg} / \mathrm{kg}$ ou $0,1 \mathrm{~mL} / \mathrm{kg}$ da solução 1:10.000, sempre infundindo bolus de 3-5 mL de soro fisiológico após a administração da medicação para ela circular e chegar até o coração, devendo-se administrar o medicamento sempre durante a massagem cardíaca. Deve-se reavaliar o ritmo e o pulso a cada dois minutos, sendo que esta reavaliação também deve incluir a efetividade da ventilação, verificando se as vias aéreas ainda permanecem pérvias e procurando tratar as causas reversíveis (lembrar dos $6 \mathrm{Hs}$ e $5 \mathrm{Ts}$ ).

\section{Caso 2}

Paciente de 7 anos, $30 \mathrm{~kg}$, internado na enfermaria de cardiologia pediátrica no pós-operatório tardio de cirurgia cardíaca, apresenta síncope com perda da consciência. É levado à sala de emergência e ao chegar lá, você encontra a criança inconsciente, em apneia, e apresentando o seguinte ritmo na monitorização cardíaca: 


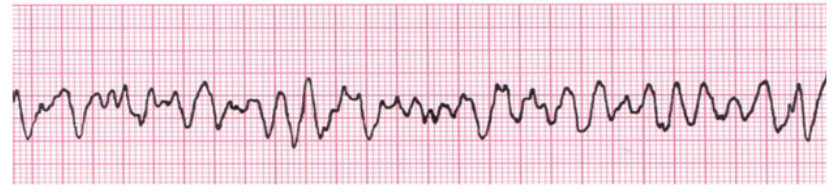

Pergunta-se: Quais são o diagnóstico e a conduta?

Resposta: O diagnóstico é fibrilação ventricular e a conduta é checar o pulso central e iniciar a massagem cardíaca, abrir as vias aéreas com posicionamento da cabeça pela colocação de coxim sob o occipício, aspirar as vias aéreas (se necessário) e fornecer oxigênio com bolsa-valva e máscara, até a chegada e o preparo do desfibrilador. Assim que ele che- gar, realiza-se um choque com $2 \mathrm{~J} / \mathrm{kg}$ e logo após, reinicia-se a RCP começando pela massagem cardíaca. Após dois minutos, verifica-se novamente o pulso central e o ritmo, e se a fibrilação ventricular persistir, prepara-se o próximo choque com $4 \mathrm{~J} / \mathrm{kg}$ e administra-se epinefrina $0,01 \mathrm{mg} / \mathrm{kg}(0,1 \mathrm{~mL} / \mathrm{kg}$ da solução $1: 10.000)$ enquanto se prepara o próximo choque, durante a massagem cardíaca ou logo após o choque, antes de iniciar a massagem. Se o ritmo persistir após 2 minutos de RCP e o segundo choque, prepara-se para o terceiro choque com $4 \mathrm{~J} / \mathrm{kg}$ e inicia-se antiarrítmico (amiodarona, lidocaína ou sulfato de magnésio). Lembrar de procurar e tratar as causas reversíveis (“6Hs e 5 Ts").

\begin{abstract}
This paper presents a review of the epidemiology, causes, diagnosis and management of cardiac arrest in children. In contrast to adults, cardiac arrest in children rarely results from a primary cardiac and sudden cause. It's usually due to respiratory failure and/or shock. Therefore, early recognition of these conditions and prompt treatment may prevent cardiac arrest and improve outcome of these patients.
\end{abstract}

Keywords: Cardiac Arrest. Child. Diagnosis. Therapeutics.

\section{Referências Bibliográficas}

1. Atkins DL, Everson-Stewart S, Sears GK, Daya M, Osmond $\mathrm{MH}$, Warden CR, et al. Epidemiology and outcomes from outof-hospital cardiac arrest in children: the Resuscitation Outcomes Consortium Epistry-Cardiac Arrest. Circulation 2009; 119: 1484-91.

2. de Mos N, van Litsenburg RR, McCrindle B, Bohn DJ, Parshuram CS. Pediatric in-intensive-care-unit cardiac arrest: incidence, survival, and predictive factors. Crit Care Med 2006; 34: 120915.

3. Berg MD, Schexnayder SM, Chameides L, Terry M, Donoghue A, Hickey RW, et al. Pediatric basic life support: 2010 American Heart Association Guidelines for Cardiopulmonary Resuscitation and Emergency Cardiovascular Care. Pediatrics 2010; 126: e1345-60.

4. Reis AG, Nadkarni V, Perondi MB, Grisi S, Berg RA. A prospective investigation into the epidemiology of in-hospital pediatric cardiopulmonary resuscitation using the international Utstein reporting style. Pediatrics 2002; 109: 200-9.

5. Donoghue AJ, Nadkarni V, Berg RA, Osmond MH, Wells G, Nesbitt L, et al. Out-of-hospital pediatric cardiac arrest: an epidemiologic review and assessment of current knowledge. Ann Emerg Med 2005; 46: 512-22.

6. Gerein RB, Osmond MH, Stiell IG, Nesbitt LP, Burns S; OPALS Study Group. What are the etiology and epidemiology of outof-hospital pediatric cardiopulmonary arrest in Ontario, Canada? Acad Emerg Med 2006; 13: 653-8.

7. Kleinman ME, Chameides L, Schexnayder SM, Samson RA, Hazinski MF, Atkins DL, et al. Pediatric advanced life support: 2010 American Heart Association Guidelines for Cardiopul- monary Resuscitation and Emergency Cardiovascular Care. Pediatrics 2010; 126: e1361-99.

8. American Heart Association, PALS - Pediatric Advanced Life Support - Provider Manual. 2006.

9. Kleinman ME, Chameides L, Schexnayder SM, Samson RA, Hazinski MF, Atkins DL, Berg MD, et al. Part 14: pediatric advanced life support: 2010 American Heart Association Guidelines for Cardiopulmonary Resuscitation and Emergency Cardiovascular Care. Circulation 2010; 122(Suppl 3): S876-908.

10. Perondi MB, Reis AG, Paiva EF, Nadkarni VM, Berg RA. A comparison of high-dose and standard-dose epinephrine in children with cardiac arrest. N Engl J Med 2004; 350: 1722-30.

11. Meaney PA, Nadkarni VM, Atkins DL, Berg MD, Samson RA, Hazinski MF, et al. Effect of defibrillation energy dose during in-hospital pediatric cardiac arrest. Pediatrics 2011; 127: e1623.

12. Field JM, Hazinski MF, Sayre MR, Chameides L, Schexnayder SM, Hemphill R, et al. Part 1: executive summary: 2010 American Heart Association Guidelines for Cardiopulmonary Resuscitation and Emergency Cardiovascular Care. Circulation 2010; 122(Suppl 3): S640-56.

13. Travers AH, Rea TD, Bobrow BJ, Edelson DP, Berg RA, Sayre MR, et al. Part 4: CPR overview: 2010 American Heart Association Guidelines for Cardiopulmonary Resuscitation and Emergency Cardiovascular Care. Circulation 2010; 122(Suppl 3): S676-84.

14. Kleinman ME, de Caen AR, Chameides L, Atkins DL, Berg RA, Berg MD, et al. Part 10: Pediatric basic and advanced life support: 2010 International Consensus on Cardiopulmonary Resuscitation and Emergency Cardiovascular Care Science With Treatment Recommendations. Circulation 2010; 122(Suppl 2):S466-515. 